\title{
Coherent 16 Quadrature Amplitude Modulation (16QAM) Optical Communication Systems
}

\author{
Fady I. El-Nahal* \\ ${ }^{1}$ Institute for Communications Engineering, Technical University of Munich, 80290 Munich, Germany
}

Received April 15, 2018; accepted June 23, 2018; published June 30, 2018

\begin{abstract}
Coherent optical fiber communications for data rates of $100 \mathrm{Gbit} / \mathrm{s}$ and beyond have recently been studied extensively primarily because high sensitivity of coherent receivers could extend the transmission distance. Spectrally efficient modulation techniques such as $M$-ary quadrature amplitude modulation (M-QAM) can be employed for coherent optical links. The integration of multi-level modulation formats based on coherent technologies with wavelength-division multiplexed (WDM) systems is key to meeting the aggregate bandwidth demand. This paper reviews coherent 16 quadrature amplitude modulation (16QAM) systems to scale the network capacity and maximum reach of current optical communication systems to accommodate traffic growth.
\end{abstract}

The surge in the transmission capacity of wavelengthdivision multiplexed (WDM) systems has resulted in a great interest in multi-level modulation formats based on coherent technologies to meet the ever-increasing bandwidth demand [1-4]. The demonstration of digital carrier phase estimation in coherent receivers has resulted in a great interest in coherent optical communications [2]. Spectrally efficient modulation techniques known from wired or wireless communication systems such as M-ary phase shift keying (PSK), quadrature amplitude modulation (QAM) and coherent optical orthogonal frequency division multiplexing (CO-OFDM) can be employed for coherent optical links [5,6]. Modulation formats with $\mathrm{k}$ bits of information per symbol can achieve a spectral efficiency of up to $\mathrm{k}$ bit $/ \mathrm{s} / \mathrm{Hz} /$ polarization compared with $1 \mathrm{bit} / \mathrm{s} / \mathrm{Hz} /$ polarization for binary modulation formats, where $\mathrm{k}$ is the number of bits per symbol. Moreover, since the phase information is preserved after detection, linear equalization methods can be used to compensate for linear optical impairments, such as chromatic dispersion and polarization mode dispersion (PMD). In addition, advanced forward error correction (FEC) techniques can be applied to increase the reach and robustness of optical communication systems. These aforementioned advantages of coherent links have a great potential to revolutionize current optical communication systems [3, 7].

Phase and polarization diverse digital coherent receivers have the potential to increase the capacity of current optical fiber networks, where all four optical carrier dimensions (the in-phase and quadrature-phase

\footnotetext{
*E-mail: fady.el-nahal@tum.de
}

components of two orthogonal polarizations) are used for modulation [8, 9]. QPSK modulation/demodulation research employing optical IQ modulation (IQM) and optical delay detection has been demonstrated where the bit rate is doubled while maintaining the symbol rate [10]. $100-\mathrm{Gb} / \mathrm{s}$ transmission systems employing quadrature phase-shift keying (QPSK) modulation, polarizationdivision multiplexing at a symbol rate of $25 \mathrm{GBd}$, have recently been demonstrated and deployed in commercial networks [11].

The recent development of high-speed digital signal processing (DSP) has offered a simple and efficient means for estimating the optical carrier phase by retrieving the IQ components of the complex amplitude of the carrier from the homodyne detected signal $[12,13]$. This concept was demonstrated in [14], where a $20-\mathrm{Gb} / \mathrm{s}$ QPSK signal was demodulated with a phase-diversity homodyne receiver followed by digital carrier-phase estimation. DSP improves system stability significantly as compared with the optical phased locked loop (OPLL) scheme [1]. Thus the integration of coherent detection and DSP has become a key part of the next generation of optical communication systems.

The post signal-processing feature of the digital coherent receiver where the IQ demodulation is a linear process is a major advantage. All the information on the complex amplitude of the optical signal is maintained even after detection $[15,16]$. Thus filtering and dispersion compensation can be performed in the electrical domain after detection. Hence dispersion compensated fiber can be excluded.

Moreover, polarization alignment can be achieved after detection as demonstrated by the polarization diversity scheme employed in the homodyne receiver [17, 18]. DSP can be used to compute and handle the complex amplitude of both horizontal and vertical polarization concurrently. Therefore, Polarization demultiplexing and compensation for Polarization mode dispersion can be achieved, hence eliminating the need for optical-polarization controllers and optical delay lines [8]. The integration of multi-level modulation formats based on coherent technologies with wavelength-division multiplexed (WDM) systems is vital to meet the aggregate bandwidth demand. One of the spectrally efficient modulation schemes is 16QAM [1]. 
This modulation format can transmit 4 bits per symbol, where each symbol is encoded by the Gray code to reduce the bit error. Hence, there is only one bit difference between any two adjacent constellation points. Moreover, we can double the bit rate using polarization-division multiplexing. This work will investigate 16QAM modulation formats to improve the system performance and increase the reach and capacity of current optical fiber links cost-effectively [9, 19-21].

The performance of a higher order modulation system can be evaluated by measuring the symbol error rate (SER) or bit error rate (BER) over a range of background noise, defined as Es/No (the ratio of energy per symbol to noise density) or $E b / N o$ (the ratio of energy per bit to noise density). For the M-QAM format, the SER is given by [22]:

$$
\begin{gathered}
S E R \approx 2\left(1-\frac{1}{\sqrt{M}}\right) \operatorname{erfc}\left(\sqrt{\frac{3}{2(M-1)} \frac{E_{s}}{N_{o}}}\right)- \\
+\left(1-\frac{2}{\sqrt{M}}+\frac{1}{M}\right) \operatorname{erfc}^{2}\left(\sqrt{\frac{3}{2(M-1)} \frac{E_{s}}{N_{o}}}\right) .
\end{gathered}
$$

A simple system for generating M-QAM transmission, adding white Gaussian noise and decoding the received symbol for various $E s / N o$ values was built using the Optisystem tool from Optiwave. The variations of SER with $E s / N o$ for M-QAM schemes is shown in Fig. 1. It is clear from the results that the SER increases as $M$ $\left(M=2^{k}\right)$ increases and there is a good agreement between the simulated and theoretical plots.

We have compared the error performance of 16QAM and 16PSK as both have the same number of constellation points. For the 16QAM format, the SER is given by:

$S E R \approx \frac{3}{2} \operatorname{erfc}\left(\sqrt{\left(E_{s} / 10 N_{o}\right)}\right)$, and for the 16PSK it is given by: $S E R \approx \operatorname{erfc}\left(\sqrt{\left(E_{s} / N_{o}\right)} \sin (\pi / M)\right)$.

The variation of SER with Es/No for both 16PSK and 16QAM is shown in Fig. 2. It is clear from the results that there is a good agreement between the theoretical and simulation results. Moreover, the 16QAM system outperforms the 16-QPSK. It can be observed from the results that for the same symbol error rate, 16QAM modulation requires around $4 \mathrm{~dB}$ lesser signal to noise ratio, when compared with $16 \mathrm{PSK}$ modulation.

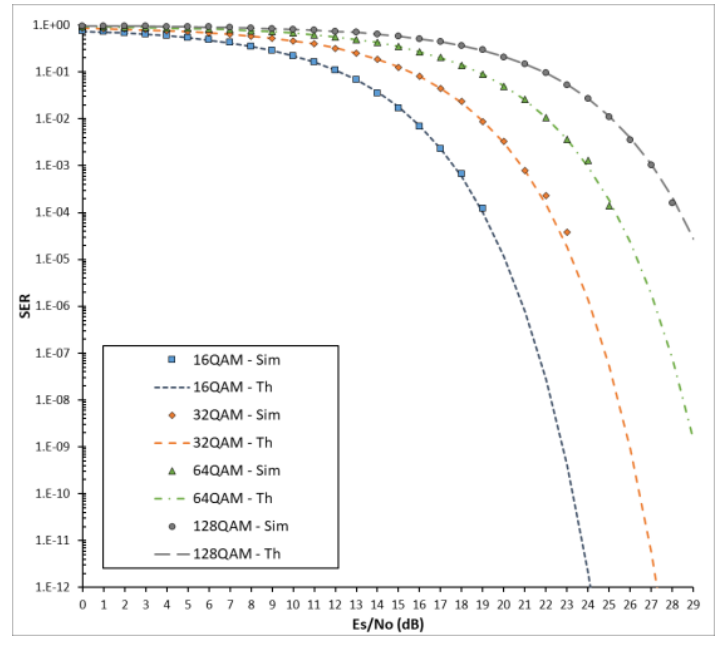

Fig. 1. SER versus Es/No for M-QAM.

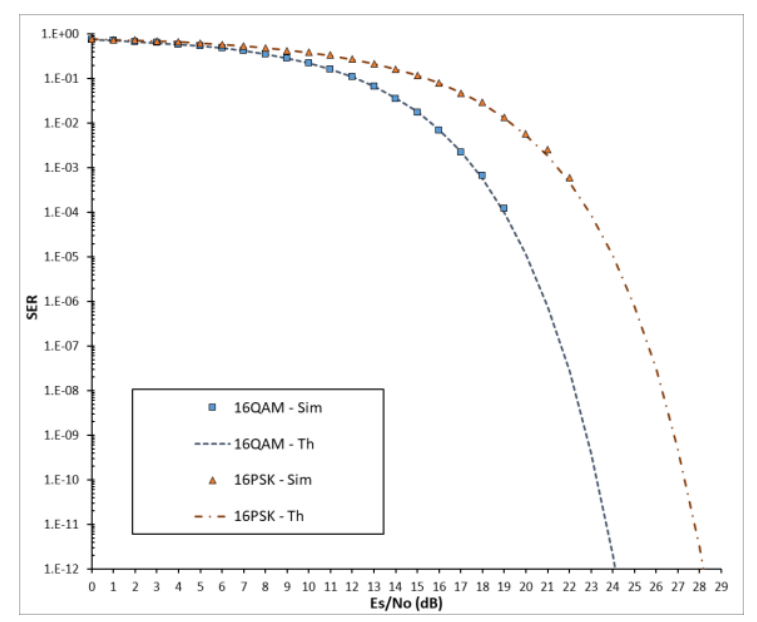

Fig. 2. SER for 16QAM and 16PSK.

Figure 3 shows the schematic of a coherent WDM dual polarization 16QAM (DP-16QAM) system. The Optical DP-16QAM transmitter generates a $112 \mathrm{~Gb} / \mathrm{s}$ DP-16QAM signal, where polarization multiplexing is used. The 1550 $\mathrm{nm}$ optical carrier signal generated by a $\mathrm{CW}$ laser is split into two orthogonal polarization components, which are modulated separately by 16QAM modulators and then combined using a polarization beam splitter (PBS). The modulated DP-16QAM signal is multiplexed by the $8 \times 1$ AWG at the transmitter and transmitted over the multi spans made up of $80 \mathrm{~km}$ of single-mode fiber (SMF), followed by Erbium doped fiber amplifier (EDFA). At the receiver, the DP-16QAM signal is de-multiplexed by the $1 \times 8$ AWG and received by the optical coherent DP16QAM receiver, which consists of a homodyne receiver design. The receiver has a local oscillator (LO) laser polarized at $45^{\circ}$ relative to the polarization beam splitter, and the received signal is separately demodulated by each LO component using two single polarization 16QAM 
receivers. Then the output of the DP-16QAM is fed to the DSP. The DSP component performs digital domain impairment compensation such as: dispersion compensation and adaptive equalization, to aid in recovering the incoming transmission signal after coherent detection. The variation of bit-error-rate (BER) with the optical signal-to-noise ratio (OSNR) at back-to-back (BT-B) and after 240-km-long SMF and the corresponding constellation diagrams of the $112 \mathrm{~Gb} / \mathrm{s}$ DP- DP-16QAM modulated signals are shown in Fig. 4. The results show that the BER decreases with the increase of OSNR and the power penalty is less than $2 \mathrm{~dB}$. Moreover, they show clear constellation diagrams, which indicates that error free transmission can be achieved.

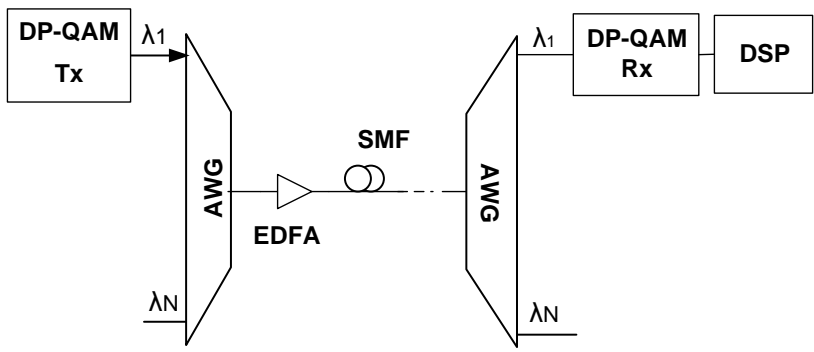

Fig. 3. Coherent WDM DP-QAM system.

In conclusions, we have investigated coherent optical communication systems based on 16QAM modulation formats. The error performance of the 16QAM receiver has been evaluated and compared with other modulation formats such as the 16PSK. The simulation results confirmed the theoretical obtained results and indicated that the 16 QAM outperformed the 16PSK system. Furthermore, a Coherent WDM dual polarization 16QAM (DP-16QAM) system has been investigated. The results show that this scheme is a practical solution for next generation coherent optical communication systems.

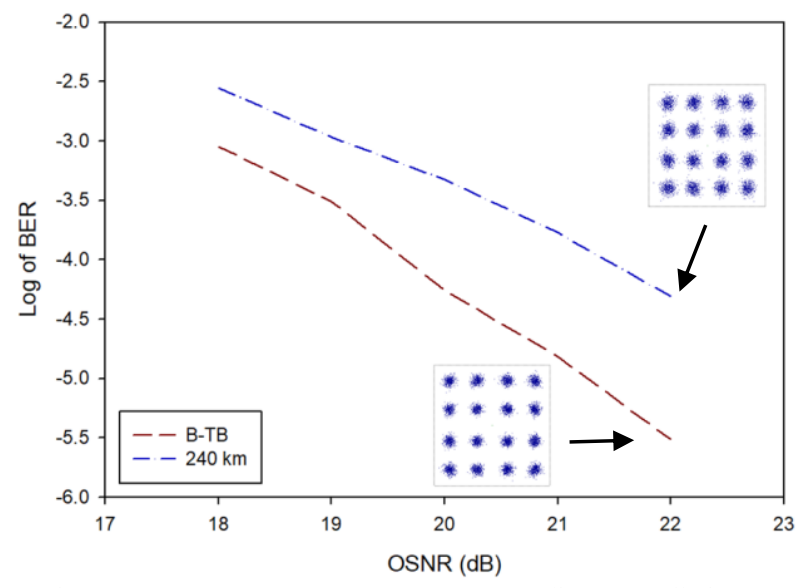

Fig. 4. BER versus OSNR. Insets: Corresponding constellation diagrams.

The author would like to acknowledge the Alexander von Humboldt Foundation, for their support.

\section{References}

[1] K. Kikuchi, J. Lightwave Technol. 34(1), 157 (2016).

[2] S. Tsukamoto, D.-S. Ly-Gagn on, K. Katoh, K. Kikuchi, Proc. OFC, Paper PDP29 (2005).

[3] K. Kikuchi, Proc. OFC, Paper Th4F.4 (2015).

[4] J.M. Kahn, K.-P. Ho, IEEE J. Sel. Topics Quantum Electron. 10(2), 259 (2004).

[5] S. Tsukamoto, K. Katoh, K. Kikuchi, IEEE Photon. Technol. Lett., 18(10), 1131 (2006).

[6] Y. Mori, C. Zhang, K. Igarashi, K. Katoh, K. Kikuchi, Opt. Expr. 17(32), 1435 (2009).

[7] H. Nakashima et al., Proc. OFC, Paper W1G.5 (2017).

[8] S.J. Savory, Opt. Expr. 16(2), 804 (2008).

[9] D.S. Millar, T. Koike-Akino, S.Ö. Arık, K. Kojima, K. Parsons, T. Yoshida, T. Sugihara, Opt. Expr. 22(7), 8798 (2014).

[10] R. Griffin, A. Carter, Proc. OFC, Paper WX6 (2002).

[11] K. Kikuchi, IEICE Electron. Expr. 8(20), 1642 (2011)

[12] F. Derr, Electron. Lett., 27(23), 2177 (1991).

[13] R. No'e, Proc. OECC, Paper 16C2-5 (2004).

[14] D.-S. Ly-Gagnon, S. Tsukamoto, K. Katoh, K. Kikuchi, J. Light. Technol. 24(1), 12 (2006)

[15] M. Taylor, IEEE Photon. Technol. Lett. 16(2), 674 (2004).

[16] S. Tsukamoto, K. Katoh, K. Kikuchi, IEEE Photon. Technol. Lett. 18(9), 1016 (2006).

[17] S. Tsukamoto, Y. Ishikawa, K. Kikuchi, Proc. ECOC, Paper Mo4.2.1 (2006).

[18] K. Kikuchi, S. Tsukamoto, J. Light. Technol. 20(13), 1817 (2008).

[19] S. Ishimura, K. Kikuchi, Proc. OFC/NFOEC, Paper M3A.2 (2014).

[20] F.I. El-Nahal, A.H.M. Husein, Int. J. Light Electron Opt. 123(14), 1301 (2012).

[21] T. Koike-Akino, D.S. Millar, K. Kojima, K. Parsons, Proc. ECOC, Paper Tu.3.C.3 (2013).

[22] B. Sklar, Digital communications: Fundamentals and Applications (Prentice-Hall, 2001). 